\title{
Cristalización de vidrios ricos en sílice preparados mediante sol-gel en el sistema alúmina-circona-sílice
}

\author{
M. POPA ${ }^{1,2}$, J. M $^{\mathrm{a}}$ CALDERÓN-MORENO 1 \\ ${ }^{1}$ Universidad Politecnica de Catalunya (UPC), ETSEIB, Dpto. Materiales, Barcelona 08028, Spain \\ ${ }^{2}$ Institute of Physical Chemistry, 77208 Bucharest, Romania
}

\begin{abstract}
La cristalización de $\mathrm{ZrSiO}_{4}$ y su evolución con la temperatura se ha estudiado en la región rica en sílice, a partir de polvos amorfos y químicamente homogéneos de alumina-sílice-circona, preparados mediante método sol-gel usando mezclas de alcóxidos. Se obtuvo un vidrio con idéntica composición mediante enfriamiento rápido por inmersión en agua del material fundido. La evolución y la microestructura de los vidrios obtenidos se estudió mediante difracción de rayos $\mathrm{X}$, infrarrojos, microscopía electrónica de barrido y análisis químico, en el rango de temperaturas hasta $1650^{\circ} \mathrm{C}$. Los materiales están formados principalmente por fase amorfa hasta $1200^{\circ} \mathrm{C}$, temperatura a la cual se observa la cristalización parcial de cristobalita. El análisis por espectroscopía de infrarrojos muestra bandas de circón en muestras tratadas térmicamente por encima de $1200^{\circ} \mathrm{C}$. Las observaciones mediante microscopía electrónica confirman la cristalización de partículas de circón y circona a $1550^{\circ} \mathrm{C}$. A $1650^{\circ} \mathrm{C}$ la cristobalita ha fundido y la única fase cristalina estable detectada mediante XRD tras 40 h a esta temperatura es circón.

Palabras clave: cristalización de circón, polvos amorfos de alumina-circona-silice, vidrios a partir de gel.
\end{abstract}

\section{Crystallization of silica-rich glasses prepared by sol-gel in the system alumina-silica-zirconia}

The crystallization of $\mathrm{ZrSiO}_{4}$ and its evolution with temperature from chemically homogeneous alumina-silica-zirconia powders prepared by sol-gel method from alcoxide mixtures was studied in the silica-rich region. A glass with the same composition was obtained by quenching in water from the melt. The gel-glasses evolution and microstructure were studied by means of XRD, IR and SEM/EDX, in the range of temperatures up to $1650^{\circ} \mathrm{C}$. The materials consisted mainly of amorphous phase up to $1200^{\circ} \mathrm{C}$, at which partial crystallization of cristobalite was observed. IR spectroscopy analysis showed zircon bands after thermal treatment at $1200^{\circ} \mathrm{C}$. The crystallization of zircon and zirconia particles at $1550^{\circ} \mathrm{C}$ was confirmed by SEM $/ \mathrm{EDX}$ analysis. At $1650^{\circ} \mathrm{C}$ the only stable crystalline phase observed after $40 \mathrm{~h}$ of thermal treatment was zircon.

Keywords: alumina-zirconia-silica; gel glassses; zircon; crystallization; amorphous powders.

\section{INTRODUCCIÓN}

Los materiales de $\mathrm{Al}_{2} \mathrm{O}_{3}-\mathrm{ZrO}_{2}-\mathrm{SiO}_{2}$ (AZS) han sido sintetizados e investigados extensamente debido a sus propiedades físico-químicas: i. e. estabilidad térmica y química, resistencia mecánica. Han encontrado aplicaciones como materiales de corte, vidrios resistentes a metales alcalinos y catalizadores heterogéneos (1). La investigación de materiales compuestos en los sistemas mencionados tiene una larga tradición. El circón $\left(\mathrm{ZrSiO}_{4}\right)$ es un importante cerámico refractario, conocido por su alta estabilidad química así como por su bajo coeficiente de expansión térmica y conductividad térmica (1-3). La cristalización de particulas de circón a partir de polvo amorfo no se conoce con detalle, i. e. hay discrepancias en la literatura sobre el mecanismo de formación de circón y los factores que afectan a su cristalización. Dependiendo de las condiciones de síntesis, se ha reportado un amplio rango de temperaturas para la formación de circón a partir de polvo amorfo (2).

Los procesos sol-gel para la formación de vidrios han ganado una gran importancia tecnológica. La estructura de los sistemas derivados de procesado sol-gel son de interés considerable por los problemas generales relacionados con la estructura de materiales amorfos y las diferencias entre los vidrios obtenidos mediante sol-gel y los obtenidos mediante enfriamiento rápido de material fundido (4). Gracias a las ventajas del uso de sol-gel para obtener materiales homogéneos a nivel molecular, hay un renovado interés en materiales del sistema ternario de óxidos AZS.
En nuestro estudio previo (5), la investigación sobre la síntesis mediante métodos químicos sol-gel y la cristalización de vidrios, tanto a partir de material fundido como derivado de geles en el sistema de óxidos ternarios AZS, determinó el método de síntesis química y la evolución microestructural de geles ternarios con la temperatura. La preparación y caracterización de los materiales se llevó a cabo para demostrar la aplicabilidad de técnicas sol-gel en la obtención de polvos amorfos homogéneos a bajas temperaturas. Precipitados homogéneos se prepararon a partir de geles.

El objetivo de la presente comunicación es el análisis de la cristalización de circón a partir del polvo amorfo, basado en difracción de rayos $\mathrm{X}$, microscopía electrónica de barrido y espectroscopía de infrarrojos en materiales AZS obtenidos mediante un método sol-gel.

\section{EXPERIMENTAL}

\subsection{Preparación de Materiales}

El procesado se llevó a cabo mediante métodos sol-gel. Los precursores fueron tetraetóxido de silicio $\mathrm{Si}\left(\mathrm{OC}_{2} \mathrm{H} \neq_{5}\right)_{4}$ TEOS (98\% Aldrich) como fuente de $\mathrm{SiO}_{2}$, $\mathrm{Al}$ trisec-butoxido $\mathrm{Al}\left(\mathrm{OC}_{4} \mathrm{H}_{9}\right)_{3}(97 \%$ Aldrich) como fuente de $\mathrm{Al}_{2} \mathrm{O}_{3}$ y $\mathrm{Zr}(\mathrm{IV})$ propóxido $\mathrm{Zr}\left(\mathrm{OC}_{3} \mathrm{H}_{7}\right)_{4}$ (70\% Aldrich) como fuente de $\mathrm{ZrO}_{2}$; se usó etanol absoluto $(\mathrm{EtOH}) \mathrm{C}_{2} \mathrm{H}_{5} \mathrm{OH}$ como 
solvente para preparar los soles y $\mathrm{HCl} 35 \%$ como catalizador. La síntesis detallada de la mezcla del gel ternario y la síntesis de los vidrios derivados del gel en el sistema AZS se presentaron previamente (5). Principalmente, el método de preparación se basó en la polimerización del alkoxisilano con otros alkóxidos para producir el gel amorfo multicomponente con red polimérica. La hidrólisis parcial del alkoxisilano introduce los grupos activos, los cuales en reacción con los otros reactantes determinan la formación de una solución polimérica (sol); la solución polimeriza con el tiempo y forma un gel rígido. Este gel es el precursor que genera, sometido a tratamiento térmico, los polvos reactivos de gel y, bajo sucesivos tratamientos térmicos, los vidrios. Estudiamos la formación de vidrios en la región rica en sílice, $\mathrm{SiO}_{2^{\prime}}$ del sistema ternario de óxidos $\mathrm{Al}_{2} \mathrm{O}_{3}-\mathrm{ZrO}_{2}-\mathrm{SiO}_{2}$. Se sintetizaron geles con composición 5-5-90 mol \% mediante método sol-gel en medio ácido y fueron tratados a temperaturas hasta $1650^{\circ} \mathrm{C}$.

\subsection{Tratamientos Térmicos}

La mezcla de alkóxidos en solución gelifica a temperatura ambiente después de 7 días y el gel resultante es blanco translúcido. El gel fue subsecuentemente secado a $60^{\circ} \mathrm{C}$. Este gel es llamado el material 'precursor'. Para analizar la cristalización en el sistema AZS, el precursor fué sometido a tratamiento térmicos en hoja de platino. Se usaron tratamientos de 1 hora a temperaturas entre $200^{\circ}$ y $800^{\circ} \mathrm{C}$, de 10 horas para tratamientos a temperaturas entre $1000^{\circ}$ y $1550^{\circ} \mathrm{C}$ y las muestras tratadas a $1650^{\circ} \mathrm{C}$ se mantuvieron a esta temperatura 40 horas para asegurar la homogenización del material.

\section{3 Caracterización}

La formación de circón (ZS) a partir del polvo amorfo se caracterizó mediante difracción de rayos X (DRX), y espectroscopía de infrarrojos (IR). La morfología de los polvos se observó mediante microscopía electrónica de barrido (MEB) y análisis de electrones electrodispersados (EDX) para determinar el contenido de Al, Si y Zr. Los patrones de difracción de rayos $\mathrm{X}$ se obtuvieron con un difractómetro D5000 Siemens $\theta-2 \theta$ usando radiación $\mathrm{Cu} \mathrm{K}_{\alpha}$ en el rango $2<\theta<65$. Los difractogramas se usaron para determinar la composición de fases, comparada con ficheros JCPDS. El espectro de IR se obtuvo con una resolución de $2 \mathrm{~cm}^{-1}$, en el rango de frecuencias desde 4000 a $400 \mathrm{~cm}^{-1}$, usando un espectrofotómetro IR (modelo Carl Zeiss Jena-Specord 75). Los espectros de infrarrojos se obtuvieron de discos finos $\left(\sim 20 \mathrm{mg} / \mathrm{cm}^{2}\right)$ de $\mathrm{KBr}$ conteniendo aproximademente $1 \%$ en peso de muestra. Las plaquetas se prepararon compactando la mezcla homogénea obtenida mediante mezcla de $\sim 1$ mg de material en $100 \mathrm{mg}$ de $\mathrm{KBr}$. La morfología del polvo se determinó en un microscopio electrónico de barrido Zeiss DSM 942 equipado con un sistema Link Energy Dispersive X-ray.

\section{RESULTADOS}

El gel obtenido en el sistema ternario usando el método sol-gel está formado por partículas amorfas, como se observa en la Fig. 1. Las fases detectadas mediante DRX a cada temperatura de tratamiento térmico se presentan en la Tabla 1.

Los tratamientos térmicos de los geles dieron polvos blancos en todos los casos. Las observaciones microestructurales mediante MEB revelaron la formación de partículas redondeadas en una matriz rica en sílice. La Fig. 2 muestra una micrografia tomada en la muestra tratada térmicamente a $1550^{\circ} \mathrm{C}$. Las partículas claras corresponden a $\mathrm{ZrO}_{2}$ (más blancas y redondeadas) y $\mathrm{ZrSiO}_{4}$ (más irregulares y en tonos grises)

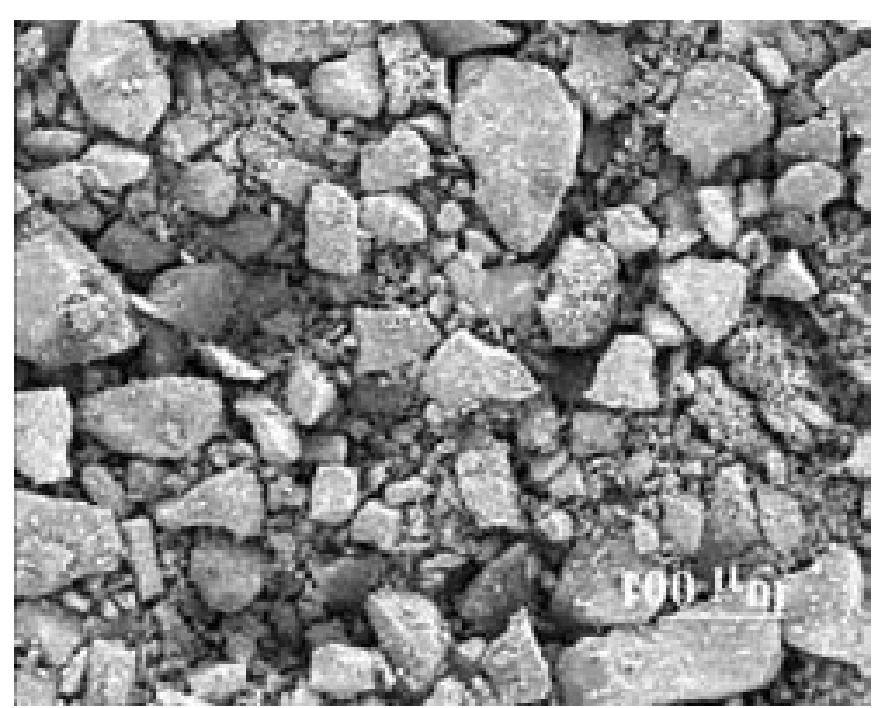

Fig. 1. Micrografía MEB del gel amorfo obtenido en el sistema ternario de óxidos.

TABLA 1. FASES DETECTADAS MEDIANTE DRX TRAS CADA TRATAMIENTO TÉRMICO.

\begin{tabular}{|c|c|}
\hline Temperatura $\left({ }^{\circ} \mathbf{C}\right)$ & Fases detectadas mediante DRX \\
\hline 1000 & amorfo \\
\hline 1200 & amorfo + trazas de cristobalita y t-circona \\
\hline 1400 & cristobalita + t-circona \\
\hline 1550 & cristobalita + circón + t-circona \\
\hline 1650 & circón + t-circona \\
\hline
\end{tabular}

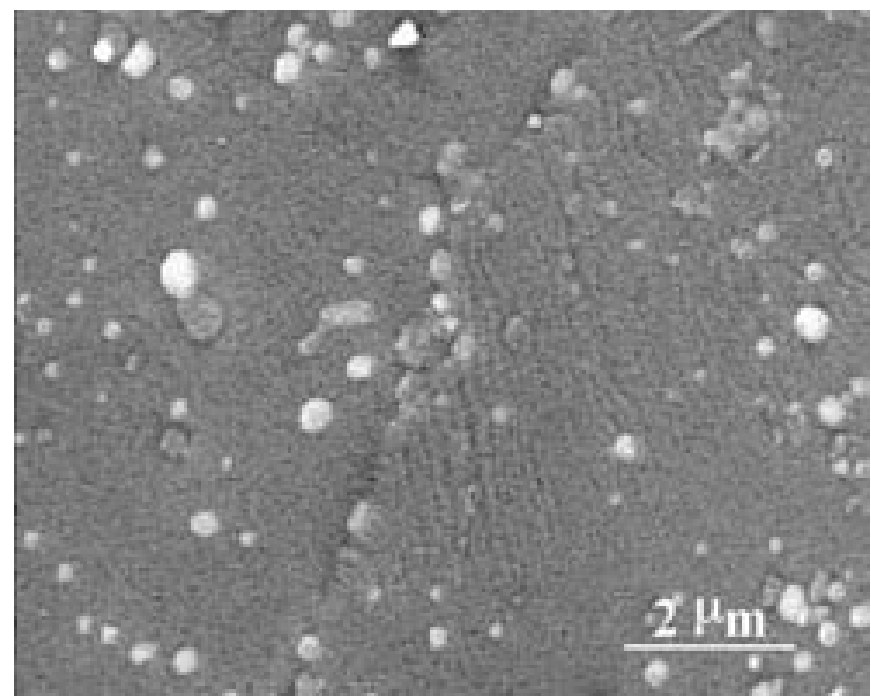

Fig. 2. Micrografía MEB mostrando el polvo AZS preparado a partir de mezclas de alcóxidos tratados a $1550^{\circ} \mathrm{C}$. Se observa la cristalización de circón (particulas irregulares de color gris) y circona (particulas redondas blancas) en la matriz de sílice más oscura.

cristalizados a partir del vidrio rico en sílice (matriz más oscura), como determinó el análisis mediante EDX. El espectro típico de EDX tomado en la matriz más oscura y en las partículas de circón se muestra en la Fig. 3. El análisis composicional indicó la ausencia de $\mathrm{Al}$ y una mayor concentration de $\mathrm{Zr}$ que en la matriz rica en sílice.

De acuerdo a los resultados de IR y DRX, las fases cristalizadas en la matriz rica en sílice son $\mathrm{ZrSiO}_{4}$ y $\mathrm{t}-\mathrm{ZrO}_{2}$ hasta $1400^{\circ}$. Se observan bandas IR de circón desde $1200^{\circ} \mathrm{C}$, pero la cristalización importante de circón ocurre a $1550^{\circ} \mathrm{C}$. Las muestras tratadas a $1650^{\circ} \mathrm{C}$ muestran la crstalización solamente de circona y circón, sin que se observe ninguna banda característica de mullita (11). 


\section{DISCUSIÓN}

En los diagramas de equilibrio de fases para el sistema binario de óxidos $\mathrm{SiO}_{2}-\mathrm{Al}_{2} \mathrm{O}_{3}$ de Aksay and Pask (12) y Klug et al (13), existe dominio de fase líquida solamente por encima de $1587^{\circ} \mathrm{C}$, mientras que para el sistema binario $\mathrm{SiO}_{2}-\mathrm{ZrO}_{2}$, la temperatura más baja de presencia de líquido es $1687^{\circ} \mathrm{C}(14)$. Observamos la aparición de líquido en las muestras tratadas a $1550^{\circ} \mathrm{C}$. Sin embargo, el patrón DRX indica que ocurre muy poca formación de líquido a esta temperatura, puesto que la fase cristobalita muestra buena cristalinidad tras el enfriamiento. Por otro lado, la muestra tratada a $1650^{\circ} \mathrm{C}$ apareció fundida casi por completo, como confirmó la amorfización completa de cristobalita observada tras el tratamiento térmico (Fig. 4). La formación de circona a temperaturas inferiores a la de disociación del circón corresponde a un proceso de no equilibrio habitual en materiales preparados mediante sol-gel. Se realizaron experiencias adicionales en mezclas de polvos con idéntica composición sometidas a tratamiento térmico a $1650^{\circ} \mathrm{C}$ durante $40 \mathrm{~h}$ y enfriadas rápidamente mediante inmersión en agua. Se detectó mediante XRD la presencia de circón como única fase cristalina.

Los resultados demuestran que la temperatura de formación de líquido en la composición ternaria está muy por debajo de la temperatura $\sim 1687^{\circ} \mathrm{C}$ del sistema binario $\mathrm{ZrO}_{2}-\mathrm{SiO}_{2}$. Estos resultados son importantes para la formación y sinterizado de cerámicos conteniendo circón y ayudan a determinar el equilibrio de fases en el sistema ternario de óxidos. Por ejemplo, Budnikov \& Litvakovskii han propuesto
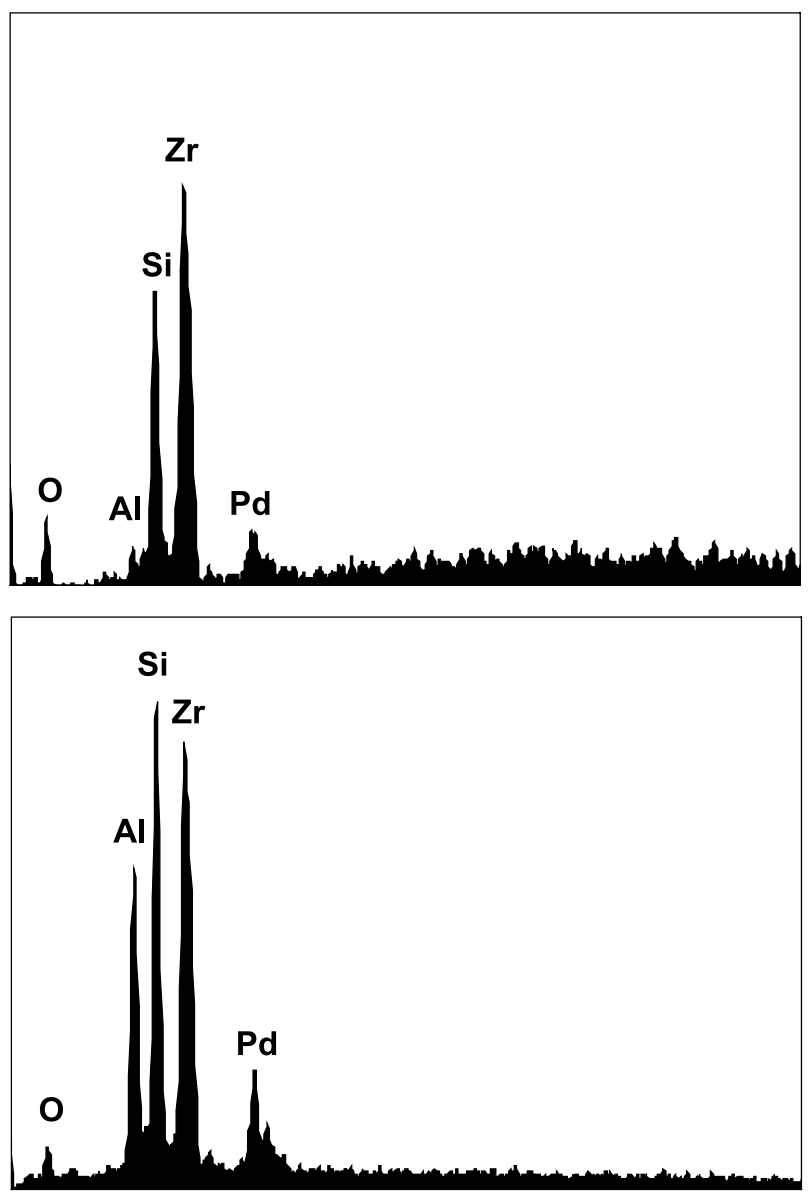

Fig. 3. Espectro de EDX obtenido en las partículas blancas de la Fig. 3 (arriba) y en la matriz más oscura (abajo). un eutéctico binario a $1545^{\circ} \mathrm{C}$ para el subsistema mullita-sílice en el sistema $\mathrm{Al}_{2} \mathrm{O}_{3}-\mathrm{SiO}_{2^{\prime}}$ mientras Greca et al la elevan a $1587^{\circ} \mathrm{C}(16)$. El sistema $\mathrm{ZrO}_{2}-\mathrm{SiO}_{2}$ muestra un eutéctico binario circón-sílice por encima de la temperatura de disociación del circón $\left(1676^{\circ} \mathrm{C}\right)$. La presencia de alúmina como una impureza común en arenas de circón causa la disminución significativa de la temperatura de aparición de fase líquida rica en sílice. La presencia de líquido está de acuerdo con la presencia de un eutéctico ternario a $1539^{\circ} \mathrm{C}$ cerca de la composición estudiada (15) calculado a partir de datos termodinámicos. Qereshi and Brett (17), Pena y De Aza (18) han señalado la significativa disminución de la temperatura de líquido en sistemas conteniendo sílice. La Fig. 5 muestra el efecto de la presencia de alúmina en las temperaturas de transformación de fase en el material. A $1650^{\circ} \mathrm{C}$ el circón coexiste como fase estable con el líquido rico en sílice, confirmando que se produce la cristalización primaria de circón a esta temperatura.

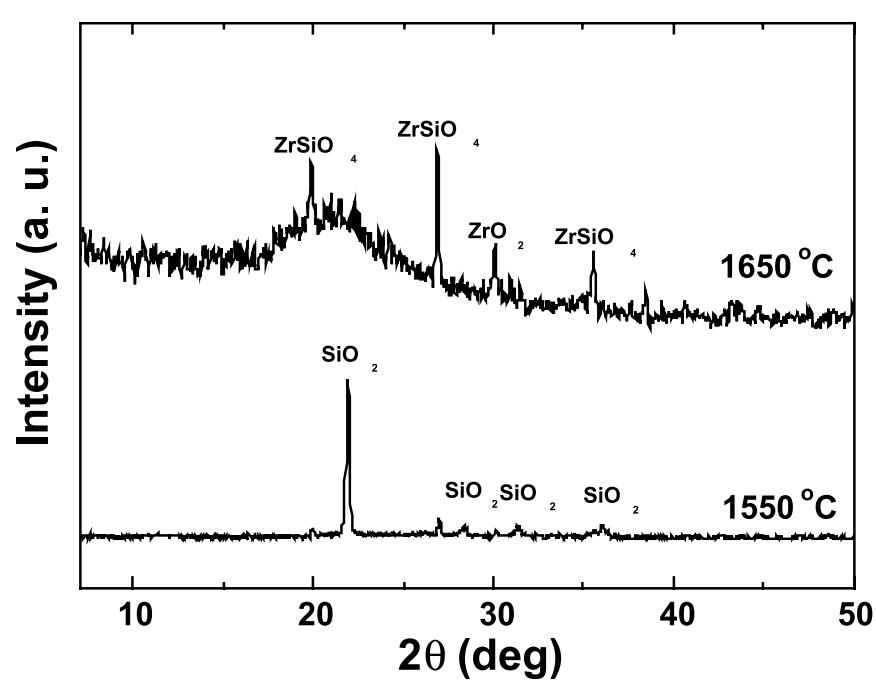

Fig. 4. Patrones de difracción de rayos $\mathrm{X}$ de las muestras tras el tratamiento térmico a 1550 y $1650^{\circ} \mathrm{C}$, mostrando la presencia de circón a ambas temperaturas y la desaparición de cristobalita a la temperatura más alta.

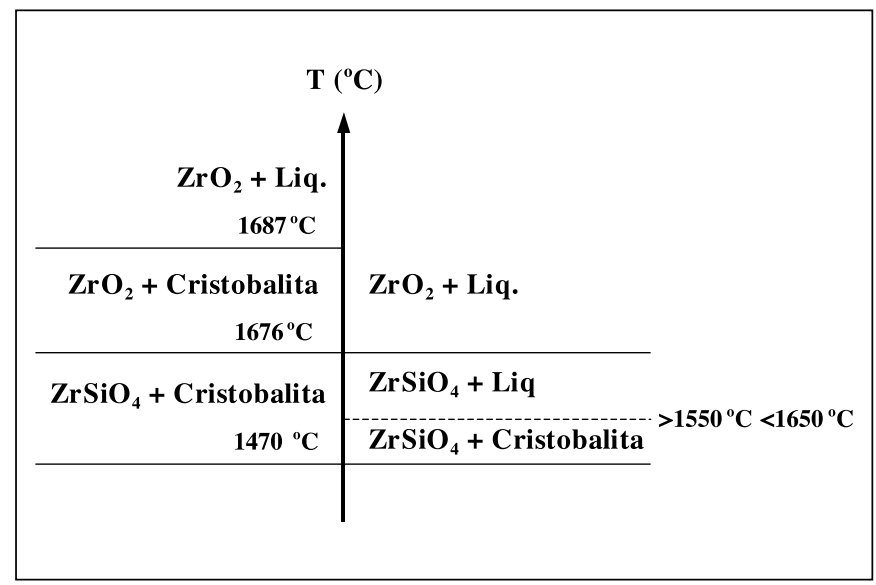

Fig. 5. Efecto de la presencia de alúmina sobre las temperaturas de transformación de fase en la composición estudiada. La temperatura de disociación del circón es superior a la nueva temperatura de líquido y el circón coexiste como fase estable con el líquido rico en sílice por debajo de $1650^{\circ} \mathrm{C}$. 


\section{CONCLUSIONES}

Se han obtenido polvos de alta pureza y químicamente homogéneos en el sistema de óxidos alumina-circona-sílice (AZS) mediante técnicas sol-gel. Los geles son amorfos hasta temperaturas de $1200^{\circ} \mathrm{C}$. En el vidrio rico en sílice se ha observado la cristalización de partículas de circón y circona tetragonal entre 1400 and $1650^{\circ} \mathrm{C}$. La cristalización de circona como fase de no equilibrio a bajas temperaturas observada es habitual en vidrios sol-gel. Geles mantenidos $40 \mathrm{~h}$. a $1650^{\circ} \mathrm{C}$ y enfriados rápidamente forman solamente circón. Se ha confirmado la cristalización primaria de partículas de circón en el caso de mezcla de polvos solidificadas por enfriamiento rápido tras tratamiento térmico prolongado a $1650^{\circ} \mathrm{C}$. La cristalización primaria de circón durante el enfriamiento desde líquido confirma el efecto de la alúmina en reducir la temperatura de formación de líquido. Como consecuencia de esta disminución observamos líquido a temperaturas inferiores a la de disociación del circón y el circón coexiste como fase estable con el líquido rico en sílice por debajo de $1650^{\circ} \mathrm{C}$. Las relaciones de estabilidad de fase en la región rica en sílice del diagrama ternario requieren estudios adicionales que estan en fase de realización.

\section{BIBLIOGRAFÍA}

1. P. Tartaj, J. Sanz, C. J. Serna, M. Ocana, “Zircon formation from amorphous spherical $\mathrm{ZrSiO}_{4}$ particles obtained by hydrolysis of aerosols". J. Mater. Sci., 1994, 29, 6533- 6538.

2. P. Tartaj, C. J. Serna, J. S. Moya, J. Requena, M. Ocana, S. de Aza, F. Guitian, "The formation of zircon from amorphous $\mathrm{ZrO} 2-\mathrm{SiO}_{2}$ powders". J.Mater. Sci., 1996, 31, 6089-6094.
3. C. Pecharroman, M. Ocana, P. Tartaj, C. J. Serna, "Infrared optical properties of zircon". Mat. Res Bull., 1994, 29 (4), 417-426.

4. S. Komarneni, "Nanocomposite Sol-gel Processing of Ceramics". J. New Ceram.,1989, 2(6) 89-94.

5. M. Popa, J. M. Calderon-Moreno, L. Popescu, M. Kakihana, R. Torrecillas, "Crystallization of gel-derived and quenched glasses in the ternary oxide $\mathrm{Al}_{2} \mathrm{O}_{3}-\mathrm{SiO}_{2}-\mathrm{ZrO}_{2}$ system". J. Non-Cryst. Solids, 2002, 297, 290-300.

6. R. M. Almeida, “Spectroscopy and Structure of sol-gel systems". J.Sol Gel Sci. \& Technol., 1998, 13, 51-59.

7. M. C. Matos, L. M. Ilharco, R. M. Almeida, "Evolution of TEOS to silica gel and glass by vibrational spectroscopy". J. Non-Cryst Solids, 1992, 147/148, 232-237.

8. S. Ricol, E. Vernaz, P. Barboux, "Synthesis of gels in the system $\mathrm{Na}_{2} \mathrm{O}-\mathrm{ZrO}_{2}$ -

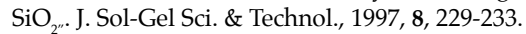

9. M. Atik, M. A. Aegerter, "Corrosion resistant sol-gel $\mathrm{ZrO}_{2}$ coatings on stainless steel”. J. Non-Cryst. Solids, 1992, 147\&148, 813-819.

10. C. J. Brinker, G. W. Scherer (eds) "Sol Gel Science. The Physics and Chemistry of Sol-gel Processing", Acad. Press, San Diego, CA, 1990

11. D. Voll, P. Angerer, A. Beran, H. Schneider, "A new assignment of IR vibrational modes in mullite". Vib. Spectr., 2002, 29, 1.

12. I. A. Aksay, J. A. Pask, "Stable and metastable equilibria in the system $\mathrm{SiO}_{2}$ $\mathrm{Al}_{2} \mathrm{O}_{3}^{\prime \prime}$. J. Am. Ceram. Soc, 1975, 58 (11-12), 507-512.

13. F. J. Klug, S. Prochazka, R. H. Doremus, J. Am. Ceram. Soc., 1987, 70 (10), 750-759.

14. W. C. Butterman, W. R. Foster, Am. Mineral., 1967, 52, 880.

15. P. Doerner, L. J. Gauckle, H. Krieg, v Lukas, G. Petzow, J. Weiss, Calphad, 1979, 3, 241.

16. M. C. Greca, J. V. Emiliano, A. M. Segadaes, J. Eur. Ceram. Soc., 1992, 9, 271-283.

17. M. H. Qereshi, N. H. Brett, Trans. Br. Ceram. Soc., 1968, 67 (6), 205-219.

18. P. Pena, S. De Aza, J. Mat. Sci., 1984, 19, 135-142.

Recibido: 01.02 .03

Aceptado: 30.11 .03 